\title{
The Research on Smartphones reliability evaluation
}

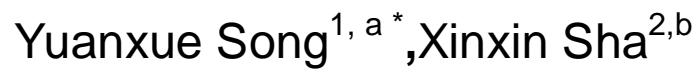 \\ ${ }^{1}$ Communication University of China, Beijing, China \\ ${ }^{2}$ Communication University of China, Beijing, China \\ asyxcuc604@163.com, bshaxinxin_cuc@126.com
}

\begin{abstract}
Keywords: Smartphone, Reliability Assessment, Software Reliability Model, Data Mining
Abstract.With the popularization of smartphone, users are more dependent on it, so the study of reliability assessment of smartphone is becoming a hot spot at present. It makes the problem more noticeable that the development of mobile phone test is slower than its production. So the demand of a reasonable solution gets more urgent. The most notable characteristics of smartphone is that it has varieties of configuration, in order to find a testing method that can be widely used, this paper presents a new idea that assessing smartphone as an integral whole. According to its function and characteristics, smartphone is divided into three parts: the hardware part, the operating system part and the application part. Then the assessment scheme and importance weight of each part are put forward on the basis of their reliability status and their influence on the whole smartphone's reliability. A reliability assessment system of smartphone is established.Besides Data Mining technology in network information and questionnaire is used to make the assessment process more complete.
\end{abstract}

\section{Introduction}

In today's information under the background of the great age of the rapid development, smart phones have not only as a kind of communication equipment, but social, education, entertainment, shopping and life information for the integration of intelligent handheld terminals. Intelligent operating system and a dual core processor application which improves the processing speed and operation performance of mobile phone, $3 \mathrm{G}$ and $4 \mathrm{G}$ networks in the development of smart phones constantly as powerful miniature handheld computers, become the most popular a kind of intelligent terminal equipment. Relying on the superiority, in just a few years the smartphone has been quite popular, its users are moving rapidly from a few people to now nearly one even more. Smartphones openness, flexibility and intelligence makes people rely on mobile phones reached an unprecedented level, but on the other hand is due to the more open mobile phone system makes its reliability is a certain degree of influence, to end users and even communication network brings a lot of unnecessary trouble, and explosive growth and the huge number of users also make smartphones reliability research is increasingly urgent.

\section{Factors affecting the reliability of the smart phone}

There are kinds of hardware testing and reliability evaluation indexes from de generation of mobile phones, and there has been a relatively complete feasible and cheap testing solutionuntil now, so the reliability of the hardware part can achieve very high degree. Once a smartphone has been put into use, at the same time its hardware already has been strictly tested, compared with the software reliability it is one or two orders of magnitude higher, therefore smartphone hardware is regarded asreliable, the assumption is reasonable.

The reliability of the operating system is mainly reflected in its and cooperation degree, and with the application of mobile phone hardware compatibility issues, so use different operating system will have an impact on the smartphone overall reliability, by comparing the differences between the various operation platform, can make a rough on the reliability of their evaluation. The existing operating system has the following several types: roughly represented by iOS/WP7 system closed source, enclosed file management system, open interface pattern; Represented by Chinese OS/WM system closed source, open file management system and the interface mode; Represented by the 
Android/Meego/WebOS/WinCE system open source, open file management system and interface mode. Openness is the principal difference between the three kinds of operating system, its reliability is also related to this, normally closed operating system because of its relatively independent and make reliability have safeguard.

The reliability of the application software for the mobile phone is vital, is also complex, analysis the reason to roughly the following several kinds: the first a mobile phone software quality is uneven, the Internet industry as an emerging industry, is in a booming period, many software vendors only pay attention to the increase of the number of users and function and does not care whether the software itself enough stable and reliable, its products are implemented is put on the market, the lack of software usability and reliability of rigorous testing and hierarchies, the existing each kind of application software quality is far gap; the second part of the smart phone users like to try a few of the applications, the low reliability of mobile application the poor stability of software failure and even system crash is more serious; the third the reliability's effect of the application software for mobile phones but also to the use of time, so different users of different habits also phone reliability have different results. Current software testing is based on the theory and method on a single software reliability research, did not relate the reliability of the application software and the mobile phone, mobile phone when use due to a software failure caused by the fault will not be able to accurately identify and promptly eliminate, so reliability assessment software to be used as a smartphone the focus of the whole reliability evaluation is very necessary. The flow chart of data processing of smart reliability evaluation model is shown in fig.1

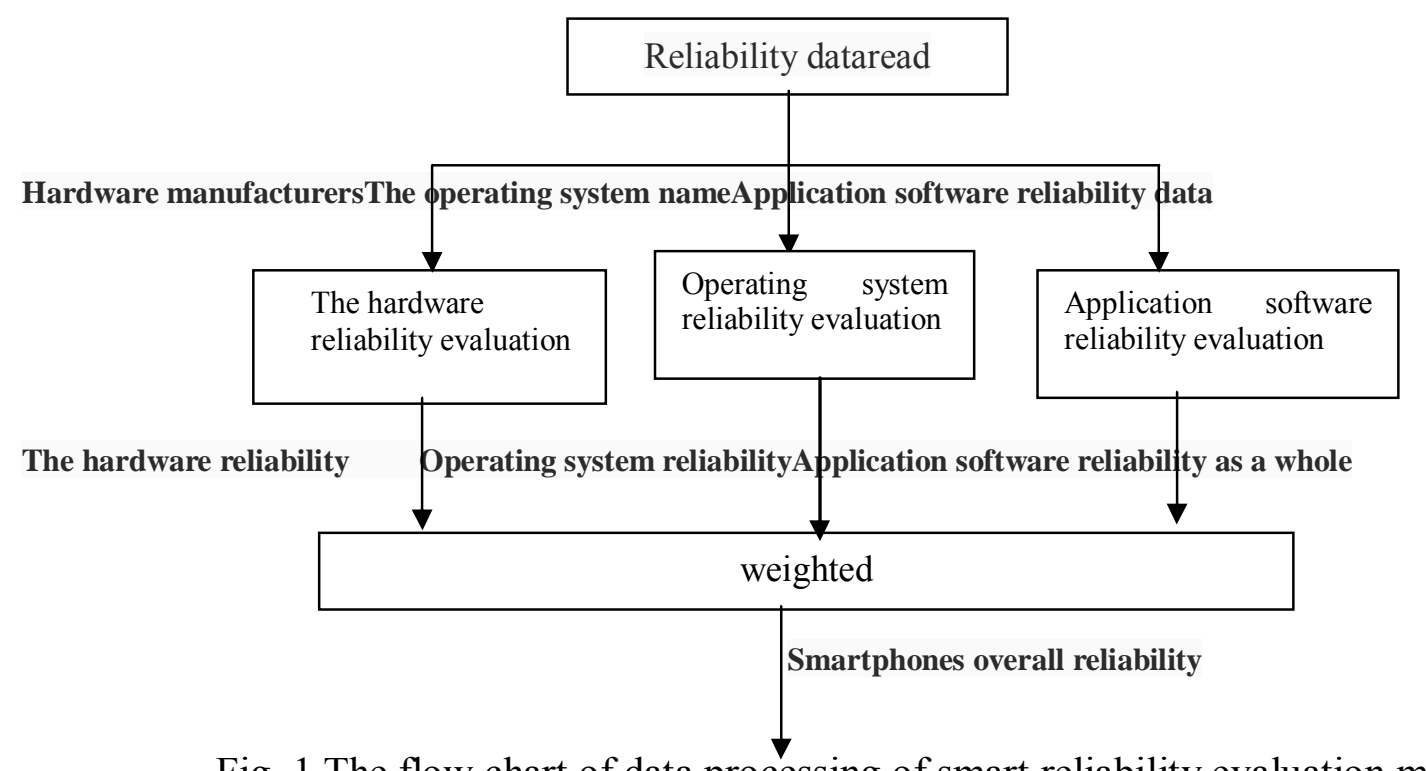

Fig. 1 The flow chart of data processing of smart reliability evaluation model

\section{The reliability of a single software}

How to get all the collection of application software in the smartphone overall reliability is an important question, also is the difficulty of this study, before that must be for each to evaluate the reliability of the software itself. This research from the three feasible aspects, realize the reliability assessment of a single application software: one is to choose the appropriate classic historical operation data collection software reliability model to evaluate calculation; Secondly, the mining software download sites on an existing user information on software quality evaluation; Three is user can set the score function, users of each software in the use of the reliability of the experience, and the grading results as a reference. In the distribution of weights, due to network data analysis and reference user ratings the two means to get reference only as the result of the reliability assessment, so as its distribution values are relatively low, are set to 0.1 ; Obtained by classical reliability model and the reliability of the results from the analysis of the application software running failure data calculation, is relatively accurate, its weight is higher, at 0.8 . 
This study chose three in the classical model reliability evaluation is fit with the experimental data and has been relatively mature and widely used in the reliability evaluation model: $\mathrm{J}$ - M model, Musa basic execution time model and G-O model. According to the failure of existing data through the model assumptions and formulas using the maximum likelihood estimation to determine the model parameters of software reliability estimate, so as to determine the concrete expression of the reliability model, thus the reliability of the application software can be predicted. Through consulting a large number of data for the three model assigned confidence weights are as follows: $0.3 \mathrm{~J}-\mathrm{M}$ model,0.4 Musa model, 0.3 G-O model. Then the reliability of the normalized results expand ten times, to make it consistent with 10-point results from other factors, so that the overall reliability of the results more intuitive.

\section{To get the algorithm of the overall reliability from all the application software}

In smartphones reliability assessment of the overall installation of the application software to include is the innovation of this study, the reliability of software applications for smart phones the influence degree of the whole reliability with the use of mobile phone users used to have very big concern, one of the most important is a user of application software, use frequency, it is clear that the user often use software reliability of the whole mobile phone impact is bigger, and some are almost do not use the software, it is installed in the phone and did not run, the impact on the overall reliability can be ignored.

An application only in actual operation the CPU resources will affect the reliability of the mobile phone, so this topic is put forward to actual use time of each application software (i.e., the execution time) for their reliability weighted, and the collection of all application software is the overall reliability of the measurement. By $\mathrm{R}_{\mathrm{app}}$ application software part of the overall reliability of it

$$
R_{a p p}=\sum_{i=1}^{N}\left(R_{i} \times \frac{t_{i}}{t_{\text {mobile }}}\right)
$$

The $\mathrm{N}$ is the total number of application software, $\mathrm{R}_{\mathrm{i}}$ is the reliability of each application, $\mathrm{t}_{\mathrm{i}}$ is the execution time of the application software.

Here it should be pointed out that the execution time of the program is really processor time used to execute the program instructions, and some applications in the background will still be a processing instruction and operation, such as data download and music playback, the time should also be counted as the execution time of the application software, this raises a new problem, namely mobile total run time will be less than the sum of all application software execution time. So when calculating the weight of each application software reliability, the denominator in the recorded total execution time instead of the mobile phone applications running total time, application of software reliability of the whole part type also make corresponding revision:

$$
R_{a p p}=\sum_{i=1}^{N}\left(R_{i} \times \frac{t_{i}}{\sum_{i=1}^{N} t_{i}}\right)
$$

Based on these theories, the flow chart of data processing of application software reliability evaluation is shown in fig. 2 


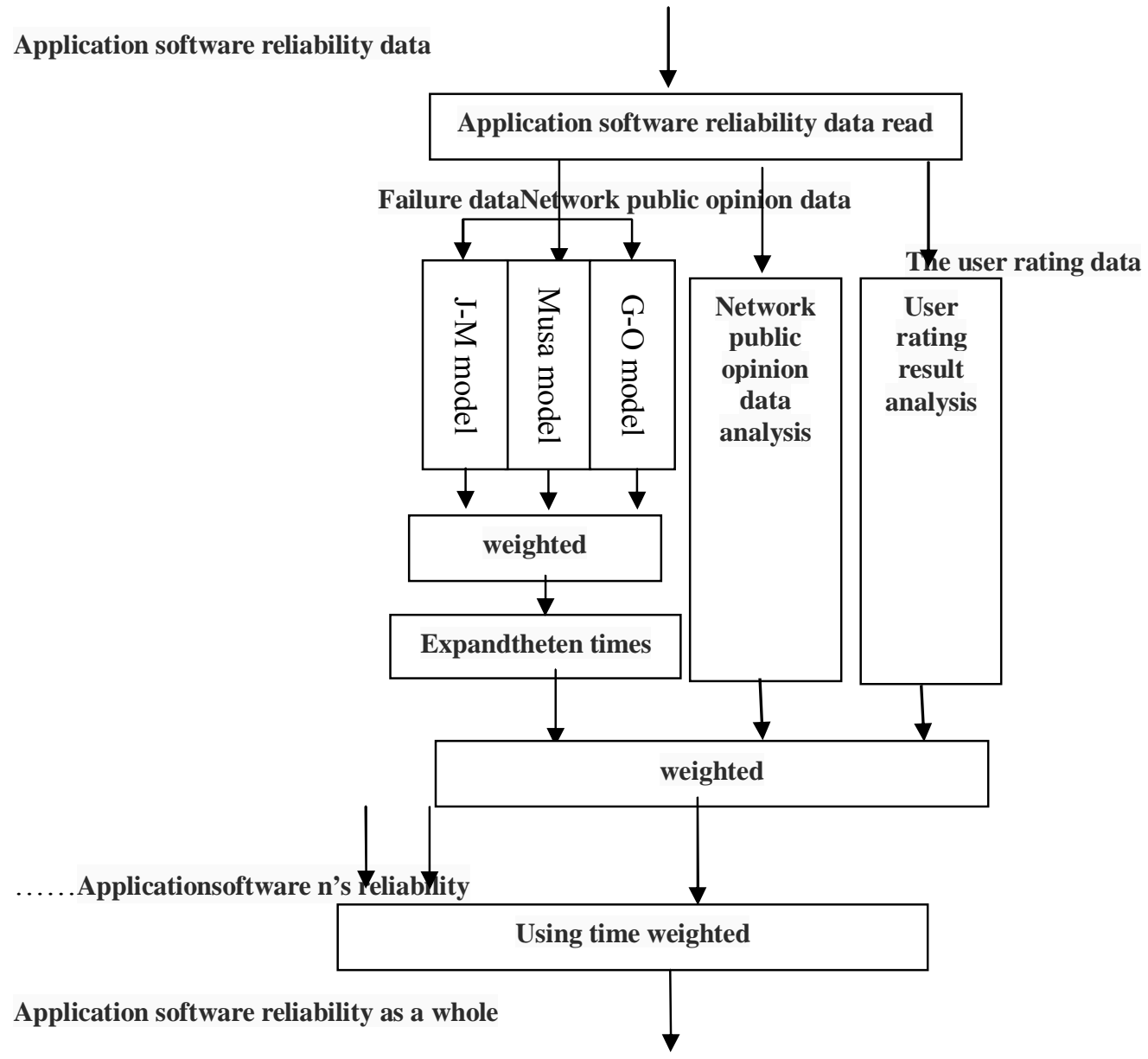

Fig. 2The flow chart of data processing of application software reliability evaluation

\section{conclusion}

This topic of the conclusion obtained is focused on the reliability of the whole smartphone evaluation ideas, specific weight distribution exactly still needs a large amount of data accurate, this can be achieved by a neural network model of training evaluation system, so as to determine the reasonable weight distribution of each part. Several limitations are put forward for details:

(1)The research just used the CPU execution time about the running time of a single application software, when the front desk to get focus on the background that is as not running, this kind of treatment method is obviously not appropriate in some procedures such as download and play music when running in the background is still CPU resources processing process, the factors should be taken into consideration.

(2) The reliability of the hardware is just roughly reference the current evaluation and market share ratio of the masses. And in most cases the embedded hardware environment on the reliability of embedded software will produce certain influence, in the study of this topic on the premise of hypothesis smartphone hardware reliability is very high, ignored the embedded hardware directly affect its software.

\section{References}

[1] Edware C Soistman , Katherine B Ragsdale . Impact of hardware and software fault on system reliability study results[R] . Martin Marietta Aerospace Orlando FL , 1988

[2] Lyu M R.Handbook of Software Reliability Engineering[M].McGraw-Hill Publishing,1995 
[3] JIA Ji-ting Design and Research of Software Reliability Model in Software Testing[J].Computer Technology and Development,2014.03 